\title{
Barriers to effective treatment of vaginal atrophy with local estrogen therapy
}

This article was published in the following Dove Press journal:

International Journal of General Medicine

14 March 2013

Number of times this article has been viewed

\author{
Suzanne Reiter \\ Mid-County Health Center, \\ Largo, FL, USA
}

Correspondence: Suzanne Reiter Mid-County Health Center, 875I Ulmerton Road, Largo, FL, USA 3377I

Tel + I 6167458463

$\mathrm{Fax}+$ I 3129519475

Email srreit2@yahoo.com
Abstract: Vaginal atrophy is a common condition among postmenopausal women, among whom many exhibit both vulvovaginal symptoms (eg, dryness, irritation, itching, and pain with intercourse) and urinary symptoms (eg, increased frequency, urgency, incontinence, urinary tract infections, and dysuria). Unfortunately, few women with symptoms of vaginal atrophy report seeking treatment from a health care provider. The goal of this article is to examine reasons why patients and health care providers do not engage in discourse regarding this important topic. It is important to initiate conversations with postmenopausal women and counsel them on both why the changes occur and potential treatment options.

Keywords: local estrogen therapy, vaginal atrophy, barriers, postmenopausal women

\section{Introduction}

Vaginal atrophy is a common condition among postmenopausal women, with up to $45 \%$ of women exhibiting symptoms. ${ }^{1,2}$ Vulvovaginal symptoms (eg, dryness, irritation, itching, and pain on intercourse) and urinary symptoms (eg, increased frequency, urgency, incontinence, urinary tract infections, and dysuria) are both common., However, only about $25 \%$ of women with symptoms of vaginal atrophy report seeking treatment from a health care provider. ${ }^{3}$ The goal of this review is to examine the reasons for this discrepancy and to suggest how to promote a dialog with premenopausal and menopausal women about age-related vaginal changes and in educating symptomatic women about their treatment options.

\section{Vaginal changes at menopause}

Before menopause, estrogen stimulates vaginal epithelial cells to produce large amounts of glycogen. ${ }^{5,6}$ These cells shed regularly into the vaginal lumen and release their stores of glycogen, which breaks down into glucose. The glucose is converted to lactic acid by the dominant microorganism, Lactobacillus, and this process maintains the vaginal $\mathrm{pH}$ at a highly acidic level of 3.5-4.5..$^{5}$ After menopause, due to decreased estrogen levels, there are fewer epithelial cells and the glycogen content of these cells is lower. Because less glucose is available for conversion to lactic acid, the vaginal $\mathrm{pH}$ rises and survival of lactobacilli is impeded, which may allow opportunistic colonization by potentially harmful and/or irritating bacteria, and consequent symptomatic infection of the vagina or urinary tract. ${ }^{5,6}$ Women often acquire bacterial vaginosis because of loss of lactobacilli. ${ }^{5,6}$ This can be problematic for women, and they may return to their health care provider repeatedly because of recurrence. 


\section{Diagnosis of vaginal atrophy}

A pH level of 5 or greater is considered consistent with vaginal atrophy, ${ }^{7}$ although a $\mathrm{pH}$ test may not typically be performed in a clinical setting. Vaginal atrophy is usually diagnosed based on patient-reported symptoms and clinician observations. Typical signs of vaginal atrophy include labial thinning, pale and dry vulvar and vaginal surfaces, a shortened and narrowed vagina with diminished rugae, and vulvovaginal erythema with or without bleeding. ${ }^{4}$ An additional quantitative measure, the vaginal maturation index, may be used to corroborate a diagnosis, but it is mainly utilized in clinical trials to demonstrate effectiveness of hormone therapy. ${ }^{4,6}$ The vaginal maturation index describes the relative proportions of parabasal, intermediate, and superficial vaginal epithelial cells, and fewer or no superficial cells indicate a low estrogen environment (Figure 1).

\section{Barriers to the discussion of postmenopausal vaginal health}

Several large recent survey-based studies have provided valuable insight into how often postmenopausal women seek and why postmenopausal women may not seek medical help for vaginal symptoms. The international "Women's Voices in the Menopause" survey was completed by 4246 women aged 55-65 years (98\% of whom were postmenopausal) living in Canada, Finland, Sweden, the United Kingdom, and the United States. ${ }^{2}$ This survey found that many women $(30 \%$ overall, 33\% in the United States) who reported experiencing vaginal discomfort (defined in the survey as dryness, smarting pain, itching, involuntary urination, or pain in the vagina in connection with touching and/or intercourse) did not talk to anyone about it, even to partners or friends. ${ }^{2}$ Only $30 \%$ of women with vaginal discomfort talked to their gynecologist (34\% in the United States), and only 29\% talked to their

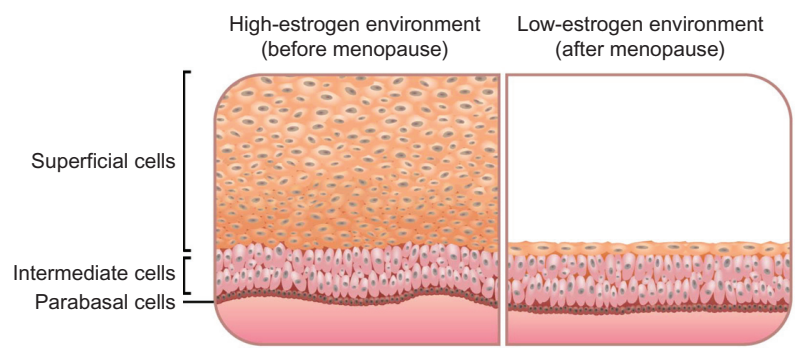

Figure I Influence of estrogen on the vaginal epithelium.

Notes: Before menopause, high levels of estrogen stimulate maturation of the vaginal epithelium to generate a thick layer of mature superficial cells. After menopause, lack of estrogen leaves the epithelium much thinner, with very few or no superficial cells. In many women, this thinning is associated with vaginal dryness, irritation, itching, and pain with intercourse, a condition referred to as vaginal atrophy or atrophic vaginitis. general practitioner (22\% in the United States). ${ }^{2}$ Reasons for not speaking to anyone about vaginal discomfort included:

- "It makes me uncomfortable/embarrassed" (60\% United States)

- "I do not think other people want to hear about my vaginal problems" (52\% United States)

- "It is private and does not concern others" (52\% United States)

- "It's just part of growing older" (49\% United States). ${ }^{2}$

A substantial number of women with vaginal discomfort who did not discuss it with anyone also expressed a preference for someone else to initiate the conversation ( $20 \%$ in the United States). ${ }^{2}$

A separate study, the REVEAL (REvealing Vaginal Effects At mid-Life) surveys,${ }^{8}$ indicated a discrepancy between patient and provider perception regarding who initiates a conversation about dyspareunia. These surveys were conducted in the United States among 1006 naturally postmenopausal women aged 45-65 years who were not taking any hormone therapy and 602 health care providers. Several key findings underscore a frequent lack of discussion about menopause-related dyspareunia:

- $44 \%$ of women experiencing dyspareunia had not discussed it with their health care provider

- Although $54 \%$ of women experiencing dyspareunia had spoken about it with their health care provider, only $10 \%$ said their health care provider had initiated this conversation; $3 \%$ either did not know or refused to answer

- In contrast, $36 \%$ of health care providers indicated that they "often" discuss vaginal pain associated with sex, while $49 \%$ indicated "sometimes"; only $14 \%$ of health care providers said "rarely" and only $2 \%$ said "never."

The REVEAL study also reported a few major barriers to care for dyspareunia (cited by women who had not talked to a health care provider about dyspareunia):

- Embarrassment (39\%)

- A belief that nothing can be done medically (26\%)

- A belief that it is not an appropriate discussion to have with a health care provider $(23 \%){ }^{8}$

More generally, this study found that $41 \%$ of the women surveyed had not spoken to anyone about their sexual health and intimacy in the past 12 months, while only $38 \%$ had spoken to a health care provider. ${ }^{8}$ These percentages differed by age group:

- Of women aged 45-49 years, $48 \%$ talked to a health care provider and $29 \%$ talked to no one

- Of women aged 50-54 years, $40 \%$ talked to a health care provider and $35 \%$ talked to no one 
- Of women aged 55-59 years, 38\% talked to a health care provider and $42 \%$ talked to no one

- Of women aged 60-65 years, 34\% talked to a health care provider and $49 \%$ talked to no one.

Along the same lines, the VIVA (Vaginal Health: Insights, Views, and Attitudes) study ${ }^{9}$ asked how women feel or would feel about speaking to their doctor about vaginal discomfort. In this study, the 3520 respondents were postmenopausal women aged 55-65 years living in Canada, Denmark, Finland, Norway, Sweden, the United Kingdom, and the United States. Important patient perspectives gained from this study were as follows:

- $53 \%$ of the women surveyed would feel comfortable discussing vaginal discomfort with their doctor, although $37 \%$ would not start the conversation or would hesitate to start it

- $34 \%$ would talk to their doctor if new symptoms developed, but $19 \%$ would prefer to try self-treatment before visiting a doctor. ${ }^{9}$

Furthermore, 50\% reported that their doctor had not broached the subject of postmenopausal vaginal health $(56 \%$ in the United States). ${ }^{9}$

An additional barrier to seeking a provider's help is the inability to pay for medical care. Many postmenopausal women in the United States do not have health insurance, ${ }^{10}$ which may prevent them from pursuing professional advice. Lack of insurance coverage may also affect their decisions related to medication, including what medication to take and how closely they adhere to the recommended treatment regimen.

\section{Treatment options for vaginal atrophy and patient perception}

\section{First-line therapies}

Vaginal moisturizers, lubricants, and continued sexual activity are recommended as first-line therapies for vaginal atrophy. ${ }^{3}$ However, not all over-the-counter moisturizers and lubricants are equal. There is often confusion over which products to use, and women may not feel comfortable asking the pharmacist about them. Vaginal moisturizers, such as Replens $^{\circledR}$ (Lil' Drug Store Products, Inc, Cedar Rapids, IA, USA), are designed to be used on a regular basis to reduce vaginal dryness. Replens has been shown to improve vaginal moisture, secretions, elasticity, $\mathrm{pH}$, and symptoms, including dryness, itching, irritation, and dyspareunia. ${ }^{11,12}$ Lubricants, which may be water-based or silicone-based, are intended to be used during sexual activity, and each class of lubricants has advantages and limitations. ${ }^{13}$ Water-based lubricants with glycerin are the most widely available and most commonly recommended, although they may dry out and become sticky or tacky during use. Water-based lubricants without glycerin may last longer, but most must be purchased at specialty stores or online. Silicone-based lubricants are also best found at specialty stores or online; these are hypoallergenic and last longer than water-based lubricants. No single product will work for every woman, so it is advisable that patients try several and see whether one works well.

\section{Local vaginal estrogen}

When first-line therapies are not adequate, local vaginal estrogen may be considered. Local vaginal estrogen therapy is effective and well tolerated, ${ }^{3}$ and it is recommended over systemic estrogen therapy when treatment of vaginal atrophy is the sole aim. ${ }^{14}$ Exogenous estrogen has several important effects on the vulvovaginal tissue, including increased blood flow, increased secretions, increased thickness of the vaginal epithelium, and reduced $\mathrm{pH}$, which may help restore healthy vaginal microorganisms and prevent infection. At a clinical level, local vaginal estrogen therapy has been shown to reduce vaginal discomfort and improve dyspareunia. ${ }^{7,15}$ Local vaginal estrogen products are available in the United States as vaginal creams, ${ }^{16,17}$ a vaginal ring, ${ }^{18}$ and a vaginal tablet. ${ }^{19}$ Low-dose, local vaginal estrogen therapies, such as the tablet, ring, and low-dose conjugated estrogen cream, have demonstrated endometrial safety in studies up to one year. ${ }^{20-22}$ The vaginal tablet has been associated with the lowest amount of systemic estrogen absorption, ${ }^{23}$ and serum estrogen levels in women who received $10 \mu \mathrm{g}$ estradiol vaginal tablets remained within the normal postmenopausal range. ${ }^{24,25}$ A new ultralow concentration vaginal estriol gel (0.005\%) in phase III development allows for a 10 -fold reduction in the amount of estrogen administered in marketed products. ${ }^{26}$ It has been shown to be safe and effective for the treatment of postmenopausal vaginal atrophy ${ }^{26}$ and may prove to be another treatment option in the future.

\section{Patient perception of local vaginal estrogen}

Despite the availability of data demonstrating that local vaginal estrogen therapy is effective, safe, and well tolerated in most women, many patients are uncertain about it. In the international VIVA study, ${ }^{9} 33 \%$ of the 3520 women surveyed had negative perceptions of local vaginal estrogen therapy, while $23 \%$ had positive perceptions and $46 \%$ did not know what perceptions to have. In the same study, $30 \%$ of women reported that they would not consider taking local vaginal 
estrogen even if they knew it was effective and capable of maintaining normal hormone levels. When respondents were asked what consequences they associated with local estrogen therapy, $27 \%$ cited the risk for breast cancer and $24 \%$ cited risk for blood clot or stroke. ${ }^{9}$ More of the women surveyed associated these risks with systemic hormone replacement therapy ( $53 \%$ and $46 \%$, respectively). In total, $49 \%$ reported that they would consider taking it, while $20 \%$ were undecided. Many women also recognized that they lacked information regarding the symptoms and treatment of vaginal discomfort; only $44 \%$ felt that enough information was available. These data suggest that a large proportion of postmenopausal women may have an incomplete understanding of the risks and benefits of local vaginal estrogen and would benefit from increased communication with their health care providers.

\section{Overcoming barriers and improving communication about treatment options Discussing midlife vaginal changes}

There is a need to educate women further about midlife vaginal changes before they occur or as they are occurring, rather than after problems have developed. When women reach 40 years of age, they are typically told about the importance of routine mammography. At this time, the first conversation with patients about changes in sexual health expected at midlife can occur. Women do not tend to think of age 40 as being "old," but this age could begin a period of transition. Some women may start experiencing perimenopausal vaginal changes around this time or soon after. ${ }^{27}$ For example, some women notice dryness and do not take any action. They may not be in a relationship and they may not be too concerned about their vaginal health; however, if these women start having sex again, they may experience irritation and bleeding.

Major barriers and suggested actions to overcome the barriers in seeking treatment are listed in Figure 2. The key element in approaching each of these challenges is open communication between the provider and the patient. The conversation should begin without assumptions about sexual activity, sexual orientation, or any other topics that would impede a partnership with the patient. Below is an example of an introduction that might be helpful in starting a conversation:

"Around midlife, we begin to check with women to see whether they are experiencing any common symptoms, such as vaginal dryness, irritation, burning after sex, or

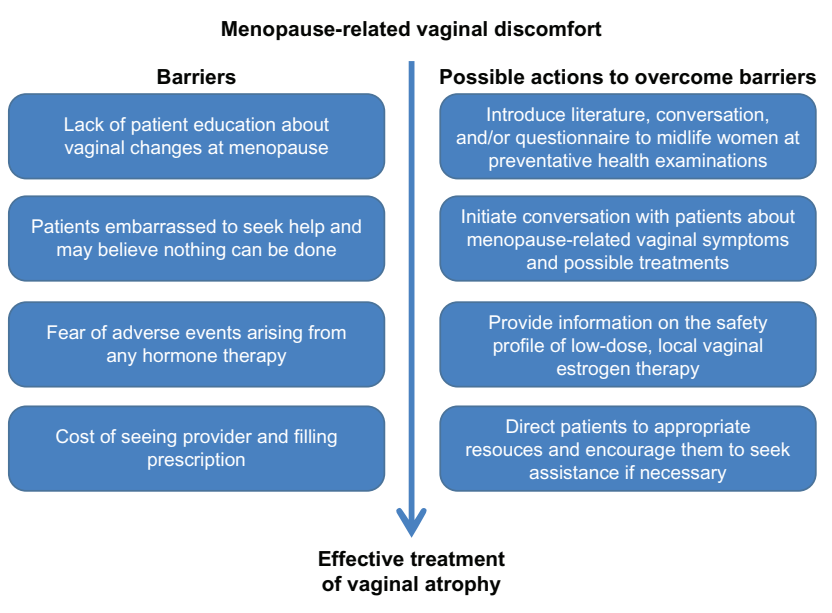

Figure 2 Barriers to the treatment of vaginal atrophy with local estrogen therapy. Notes: Several major barriers may prevent women from seeking help for menopause-related vaginal discomfort. Communication and education are key factors in overcoming these barriers.

itching. Have you noticed any symptoms like this?" If the patient responds in the affirmative, say to her, "Please tell me about what you are experiencing. Perhaps I can help you."

As shown here, it might be helpful to preface questions with a brief description of what is normal at midlife, because this can encourage women to voice their concerns. Women may also appreciate educational tools (eg, information on vaginal health with a question-and-answer form regarding symptoms to be filled out by the patient) or a concise explanation of the causes and effects of vaginal discomfort around and after menopause.

\section{Discussing treatment options}

For women who report vaginal symptoms and in whom physical examination confirms evidence of vaginal atrophy, a conversation about recommended treatment options is appropriate. Some women may hesitate to accept local vaginal estrogen because of fear of negative side effects. Women with moderate to severe vaginal atrophy and no contraindications to local vaginal estrogen may find a discussion of the benefits of minimal systemic absorption useful. Below is an example of an explanation that might help a patient put the amount of estrogen she would be receiving into context:

"Local vaginal estrogen is administered and can be effective in very low doses because it is delivered right to the tissue where it is needed - that is, the vagina. The amount of estrogen that might be taken for hot flashes, for instance, is much higher. One standard oral dose of estrogen 
(1 mg estradiol) contains the same amount of estrogen as 100 doses of the vaginal tablet, which would treat a patient for about a year."28

Providers should be prepared to offer simple explanations of the current safety and effectiveness data for available therapies.

In addition, the practical aspects of each treatment may be discussed. The $10 \mu \mathrm{g}$ estradiol tablet is administered daily for 2 weeks, then twice weekly for maintenance, ${ }^{19}$ while the conjugated estrogen cream may be administered daily in a cyclic manner ( 21 days on/7 days off) or twice weekly from the beginning. ${ }^{17}$ The estradiol cream is administered daily for $1-2$ weeks, then gradually decreased to half the initial dose over a similar period, followed by a maintenance regimen of $1-3$ times per week. ${ }^{16}$ Finally, the estradiol ring is inserted and left in place for 90 days, after which it is removed and a replacement is inserted. ${ }^{18}$

It is important to counsel patients that it may take 2 weeks or longer after initiation of local estrogen therapy to prime the vaginal epithelium and produce noticeable results (eg, relief of vaginal dryness, irritation, itching, and pain on intercourse). Also, because vaginal atrophy is a chronic condition, health care providers must consider an appropriate duration of treatment. North American Menopause Society recommendations state that local estrogen therapy should be continued as long as patients have symptom-related discomfort. ${ }^{3}$ Treatment decisions should be based on the unique circumstances that dictate benefits and risks for each patient.

\section{Summary}

Vaginal atrophy is a common condition in postmenopausal women, yet many women do not seek treatment from a health care provider. Preventative health examinations before and around menopause may be appropriate forums for initial discussion of some of the vaginal changes that may be expected when estrogen levels decrease. With further education about menopause-related vaginal changes, women experiencing vaginal discomfort may become more willing to seek help and be better able to make informed decisions regarding treatment.

\section{Acknowledgment}

Writing support was provided by Pamela Barendt of ETHOS Health Communications, Newtown, PA, USA, with financial assistance from Novo Nordisk Inc, Princeton, NJ, USA, in compliance with international guidelines for Good Publication Practice.

\section{Disclosure}

The author received no remuneration of any kind for the development of this manuscript and reports no conflict of interest in this work.

\section{References}

1. Santoro N, Komi J. Prevalence and impact of vaginal symptoms among postmenopausal women. J Sex Med. 2009;6(8):2133-2142.

2. Nappi RE, Kokot-Kierepa M. Women's voices in the menopause: results from an international survey on vaginal atrophy. Maturitas. 2010;67(3):233-238.

3. The North American Menopause Society. The role of local vaginal estrogen for treatment of vaginal atrophy in postmenopausal women: 2007 position statement of The North American Menopause Society. Menopause. 2007;14(3):357-369.

4. Sturdee DW, Panay N; on behalf of the International Menopause Society Writing Group. Recommendations for the management of postmenopausal vaginal atrophy. Climacteric. 2010;13(6): 509-522.

5. Stika CS. Atrophic vaginitis. Dermatol Ther. 2010;23(5):514-522.

6. MacBride MB, Rhodes DJ, Shuster LT. Vulvovaginal atrophy. Mayo Clin Proc. 2010;85(1):87-94.

7. Simon J, Nachtigall L, Gut R, Lang E, Archer DF, Utian W. Effective treatment of vaginal atrophy with an ultra-low-dose estradiol vaginal tablet. Obstet Gynecol. 2008;112(5):1053-1060.

8. Wyeth. REVEAL REvealing Vaginal Effects At mid-Life: Surveys of postmenopausal women and health care professionals who treat postmenopausal women. Madison, NJ: Wyeth; 2009. Available from: http://www.revealsurvey.com/pdf/reveal-survey-results.pdf. Accessed February 21, 2013.

9. Nappi RE, Kokot-Kierepa M. Vaginal Health: Insights, Views and Attitudes (VIVA) - results from an international survey. Climacteric. 2012;15(1):36-44.

10. Prickett KC, Angel JL. The new health care law: how will women near retirement fare? Womens Health Issues. 2011;21(5):331-337.

11. Nachtigall LE. Comparative study: Replens versus local estrogen in menopausal women. Fertil Steril. 1994;61(1):178-180.

12. Bygdeman M, Swahn ML. Replens versus dienoestrol cream in the symptomatic treatment of vaginal atrophy in postmenopausal women. Maturitas. 1996;23(3):259-263.

13. Andelloux M. Products for sexual lubrication: understanding and addressing options with your patients. Nurs Womens Health. 2011; 15(3):253-257.

14. The North American Menopause Society. The 2012 Hormone Therapy Position Statement of The North American Menopause Society. Menopause. 2012;19(3):257-271.

15. Suckling J, Lethaby A, Kennedy R. Local oestrogen for vaginal atrophy in postmenopausal women. Cochrane Database Syst Rev. 2006;4:CD001500.

16. Estrace ${ }^{\mathrm{TM}}$ (estradiol vaginal cream, USP, $0.01 \%$, prescribing information). Rockaway, NJ: Warner Chilcott; 2011.

17. Premarin ${ }^{\mathrm{TM}}$ (conjugated estrogen, vaginal cream, prescribing information). Philadelphia, PA: Wyeth Pharmaceuticals Inc; 2010.

18. Estring ${ }^{\mathrm{TM}}$ (estradiol vaginal ring, prescribing information). New York, NY: Pharmacia and Upjohn Company; 2008.

19. Vagifem ${ }^{\mathrm{TM}}$ (estradiol vaginal tablets, prescribing information). Princeton, NJ: Novo Nordisk Inc; 2009.

20. Simon J, Nachtigall L, Ulrich LG, Eugster-Hausmann M, Gut R. Endometrial safety of ultra-low-dose estradiol vaginal tablets. Obstet Gynecol. 2010;116(4):876-883. 
21. Weisberg E, Ayton R, Darling G, et al. Endometrial and vaginal effects of low-dose estradiol delivered by vaginal ring or vaginal tablet. Climacteric. 2005;8(1):83-92.

22. Bachmann G, Bouchard C, Hoppe D, et al. Efficacy and safety of low-dose regimens of conjugated estrogens cream administered vaginally. Menopause. 2009;16(4):719-727.

23. Eugster-Hausmann M, Waitzinger J, Lehnick D. Minimized estradiol absorption with ultra-low-dose $10 \mu \mathrm{g} 17 \beta$-estradiol vaginal tablets. Climacteric. 2010;13(3):219-227.

24. Lee JS, Ettinger B, Stanczyk FZ, et al. Comparison of methods to measure low serum estradiol levels in postmenopausal women. J Clin Endocrinol Metab. 2006;91(10):3791-3797.

25. Santen RJ, Demers L, Ohorodnik S, et al. Superiority of gas chromatography/tandem mass spectrometry assay (GC/MS/MS) for estradiol for monitoring of aromatase inhibitor therapy. Steroids. 2007; 72(8):666-671.
26. Cano A, Estevez J, Usandizaga R, et al. The therapeutic effect of a new ultra low concentration estriol gel formulation $(0.005 \%$ estriol vaginal gel) on symptoms and signs of postmenopausal vaginal atrophy: results from a pivotal phase III study. Menopause. 2012;19(10): 1130-1139.

27. Cumming GP, Currie HD, Moncur R, Lee AJ. Web-based survey on the effect of menopause on women's libido in a computer-literate population. Menopause Int. 2009;15(1):8-12.

28. Panay N, Maamari R. Treatment of postmenopausal vaginal atrophy with $10-\mu \mathrm{g}$ estradiol vaginal tablets. Menopause Int. 2012;18(1): 15-19.

\section{Publish your work in this journal}

The International Journal of General Medicine is an international, peer-reviewed open-access journal that focuses on general and internal medicine, pathogenesis, epidemiology, diagnosis, monitoring and treatment protocols. The journal is characterized by the rapid reporting of reviews, original research and clinical studies across all disease areas.
A key focus is the elucidation of disease processes and management protocols resulting in improved outcomes for the patient. The manuscript management system is completely online and includes a very quick and fair peer-review system. Visit http://www.dovepress.com/ testimonials.php to read real quotes from published authors.

Submit your manuscript here: http://www.dovepress.com/international-journal-of-general-medicine-journal 